\title{
Managing Diabetes in the COVID-19 Era: Focus on COVID-19 Safe Clinics, Telemedicine, and Diabetes Self-Management
}

\author{
Rajan Palui ${ }^{1}$ Subhodip Pramanik ${ }^{2}$ Arindam Ray ${ }^{3}$ \\ ${ }^{1}$ Department of Endocrinology, The Mission Hospital, Durgapur, \\ West Bengal, India \\ 2Department of Endocrinology, Neotia Getwel, Siliguri, \\ West Bengal, India \\ ${ }^{3}$ Department of General Medicine, College of Medicine and \\ Sagore Dutta Hospital, Kolkata, West Bengal, India \\ ${ }^{4}$ Department of Endocrinology, Medical Super Speciality Hospital, \\ Kolkata, West Bengal, India \\ ${ }^{5}$ Department of General Medicine, IPGMER, Kolkata, West Bengal, \\ India \\ ${ }^{6}$ Department of Endocrinology, CEDAR Superspeciality Clinics, \\ New Delhi, India
}

Uttio Gupta ${ }^{4}$ Sattik Siddhanta ${ }^{5}$ Deep Dutta ${ }^{6}$

J Soc Health Diab:2020;8:3-7

\begin{abstract}
Novel coronavirus disease 2019 is a dreaded pandemic that is going to persist, and diabetic people with poor glycemic control are at high risk of complications and death from it. Social distancing being the only way to prevent infection spread, it is very difficult to continue usual clinic-based diabetes care. So, in this review, we focus

Keywords

- coronavirus

- lockdown

- telemedicine to discuss safe clinic practice recommendations, scope of telemedicine in care of follow-up patients, and diabetes self-management that every individual with diabetes needs to follow. These issues will be of utmost important as we need to understand and implement personal and social hygiene in a better way even in post lockdown period.
\end{abstract}

\section{Introduction}

Novel coronavirus disease 2019(COVID-19), caused by infection with severe acute respiratory syndrome coronavirus 2 , is a dreaded pandemic sweeping across the globe since the beginning of 2020. With no definitive treatment or vaccine available to decrease the associated morbidity and mortality, social distancing is the only effective tool till date to curtail the spread of infection, resulting in a lesser burden of severely ill patients at a point of time, thus preventing the collapse of health-care infrastructure in a particular country. This is especially important for a developing country like India, where the health-care infrastructure is already overstretched to the limits.

An unrealized side effect of the COVID-19 pandemic is further restriction of access to health care by people living with noncommunicable diseases like diabetes, hypertension, and cardiovascular disease. Due to lack of transport, most of the patients cannot visit the hospitals for care. Also, even if a patient is able to reach a hospital, chances are that a significant number of hospitals, especially in the private health-care setup, have curtailed their routine outpatient services due to the pandemic, hence again preventing the patient from access to proper timely health care. Further it must be remembered that it is the very patients living with diabetes, hypertension, and cardiovascular disease are more likely to have complications due to COVID-19 infection, resulting in a greater mortality. Hence, these are the very patients who need to practice social distancing most religiously to prevent the infection in the first place. This also makes people living with these noncommunicable diseases more hesitant to leave homes, even if they have valid health issues, which would warrant an intervention. This article highlights the role of telemedicine in ameliorating this risk and ensuring optimal and timely delivery of health-care services.
DOI https://doi.org/ $10.1055 / \mathrm{s}-0040-1716439$ ISSN 2321-0656. (c) 2020. Novo Nordisk Education Foundation.

This is an open access article published by Thieme under the terms of the Creative Commons Attribution-NonDerivative-NonCommercial-License, permitting copying and reproduction so long as the original work is given appropriate credit. Contents may not be used for commercial purposes, or adapted, remixed, transformed or built upon. (https://creativecommons.org/licenses/by-nc-nd/4.0/).

Thieme Medical and Scientific Publishers Pvt. Ltd. A-12, 2nd Floor, Sector 2, Noida-201301 UP, India 


\section{Association of Diabetes and COVID-19}

Among 72,314 cases, diabetes subjects had mortality rate of $7.3 \%$ compared with nondiabetic subjects who had $2.3 \%$ as reported by Wu and McGoogan. ${ }^{1}$ Yang et al also confirmed that the most distinctive comorbidities of COVID-19 were cerebrovascular diseases (22\%) and diabetes (22\%) and documented 32 nonsurvivors from a group of 52 intensive care unit patients. ${ }^{2}$ In another study, comorbidities of hypertension (23.7\%), diabetes mellitus (16.2\%), coronary heart diseases (5.8\%), and cerebrovascular disease (2.3\%) documented in 1,099 patients with confirmed COVID-19. ${ }^{3}$

\section{How to Make a Clinic COVID-19 Compliant to Prevent the Spread of COVID-19 among Health-Care Staff and Patients?}

Social distancing remains the cornerstone in tackling COVID-19 pandemic. For all practical purposes, patients may be requested to avoid or postpone routine visits if possible. Patients suffering from minor ailments may be advised to visit local hospital or consult via telephonic conversation or mail rather than personal visit. However, the following precautions must be maintained for smooth and proper functioning of a diabetic clinic in this crisis time of COVID-19 pandemic. First one must ensure that only the patient and a maximum of one attendant may be allowed to enter the clinic. One must also ensure that they have opened their footwear before entering. One must ensure that both of them are wearing masks and maintaining a distance of at least $1 \mathrm{~m}$ between themselves and $2 \mathrm{~m}$ distance with the consultant. They should sign a written declaration that neither of them is having fever, cough, or sore throat at the time of visit. Both the doctor and patient should ideally rub their hands with soap and water or sanitizers both before and after consultation of each patient. Keep a transparent screen between the patient and the consultant. One must wipe the diaphragm of stethoscope or sphygmomanometer after each and every consultation. Do not see patients continuously, please keep 1 minute in hand between each patient for proper personal hand hygiene and also stethoscope hygiene. Avoid handshake or touching your face, nose, and eyes unnecessarily. Sanitize your hands after checking the reports and previous prescriptions brought by the patient. Ask for any recent history of travel to every patient. Use disposable gloves and face shield whenever necessary. Windows should be kept open with cross-ventilation. Try to avoid air conditioners or coolers. Restrict use mobile phones before hand hygiene, disinfect phone with hand rub. Try to use headphones and ear pods to minimize contact with mobile phone. Disinfect all the patient area every 2 hours (including tables, chairs, side rails, lift, door handles, and paperweights) by a dedicated staff who knows proper disinfection procedures. Wash hands after returning home, undress and wash all the apparels used in the clinic immediately and separately. Educate patients about social distancing, hand hygiene, cough etiquette, and proper use of masks. Try to put up posters or pamphlets about DO's and DON'T'S to increase the awareness regarding COVID-19. ${ }^{4}$

\section{Is Telemedicine the Way Forward?}

Yes, indeed telemedicine is the way forward. But as with everything in life, it is important to realize that not all patients would be benefitted by telemedicine. This review helps to classify and define the cohort of people living with diabetes, who are most likely to be benefitted by telemedicine. Also, we highlight the scenarios, where telemedicine would not have significant impact on the patient health, and would need urgent physical examination and care by the doctor.

\section{When Would Telemedicine Be Helpful in Diabetes Care?}

- More than $90 \%$ of people living with diabetes is type 2 diabetes mellitus (T2DM), which is primarily a lifestyle disorder. T2DM is all about self-management and self-care. Telemedicine has a major role in educating patients how to self-manage T2DM, how to improve diet and lifestyle.

- Treating doctors can shoot videos about healthy diet and lifestyle practices and share with patients. Fine tuning of individualized diet and lifestyle practices can be done by the doctor with the patient through telemedicine.

- Telemedicine videos and consults can be used to teach correct insulin injection, use and storage practices.

- Telemedicine can be used to empower patients how to do self-monitoring of blood glucose (SMBG) at home and to self-diagnose and treat mild hypoglycemia.

- In patients on oral diabetes medications, telemedicine can bring the patient and the doctor together, where the treatment regimen can be fine-tuned, based on the home SMBG charts, so that the patient has minimal to no hypoglycemic and hyperglycemic spikes (glycemic variability).

- Nearly $50 \%$ of people living with diabetes have coexisting hypertension. Telemedicine can be used to optimize antihypertension medication based on the home blood pressure monitoring charts.

- Telemedicine can be used to educate the patients about the major end-organ damage related to diabetes (coronary artery disease, stroke/cerebrovascular accidents/ nephropathy/neuropathy/retinopathy/peripheral vascular disease). If patients develop any of the related high-risk symptoms, the patients should not waste time at home, and should seek emergent medical care.

- In a stable, ambulatory person living with diabetes without any major comorbidities, the patient can get home blood sampling done. Most of such patients need 3 to 6 months hemoglobin A1c (HbA1c), and annual lipid profile, renal function test, spot urine albumin creatinine ratio, and liver profile. The soft copies of the report can be shared with the treating doctor and the treatment can be modulated through telemedicine. 


\section{When Should Telemedicine Be Avoided in Diabetes Care?}

Following are the scenarios where the patients need physical examination by the doctor for optimal care. Trying to use telemedicine can lead to wastage of precious time, which may impair the outcomes in certain scenarios.

- In a person living with type 1 diabetes mellitus (T1DM), who becomes unconscious, it may likely due to severe hypoglycemia or an hyperglycemic crisis like diabetic ketoacidosis. Such patients need hospital care to find and treat the precipitating cause, adequate intravenous hydration, and modulation of insulin therapy.

- Respiratory symptoms in people living with diabetes would warrant a clinical evaluation and testing if needful.

- Chest pain, especially a typical cardiac chest pain, warrants at least electrocardiogram and clinical examination (NB: a large number of cardiac events in diabetes are clinically silent).

- People in extremes of age, who have a lower threshold of metabolic decompensation.

- Patients with persisting fever more than 3 to 4 days would further require detailed examination and evaluation.

- Any patient who is so ill that he or she not able to take care of himself and do his daily chores at home.

The recent telemedicine practice guideline (Indian Medical Council [Professional Conduct, Etiquette and Ethics] [Amendment] Regulations, 2020) ${ }^{4}$ allows all registered medical practitioners to provide telemedicine service to any patients from any part of India. However, all the medical professionals have to undergo a mandatory online course within 3 years of its notification to ensure that professional and ethical standards are maintained. The telemedicine consultation can be text (synchronous or asynchronous), audio-or video-based, and can be between medical practitioners and patients, caregivers or health workers. If the patient has initiated the teleconsultation, then the consent is implied but otherwise explicit consent is needed from patient. There are specific restrictions on the type of medications that can be prescribed by telemedicine depending upon the modes (text/ audio/video) and types (first or follow-up) of teleconsultation. Medicine included in schedule X of drug and cosmetic act and narcotics cannot be prescribed by telemedicine.

\section{Tips for Diabetes Self-Management in the Era of COVID-19}

Diet $^{5}$

Healthy nutrition is an essential component of diabetes management specially in this lockdown phase. It is therefore imperative for people with diabetes to eat a varied and balanced diet to keep their blood glucose levels stable and enhance their immune system. It is recommended to:

- Give priority to foods with a low glycemic index (e.g., vegetables, whole wheat pasta/noodles).

- Avoid excessive consumption of fried foods.
- Limit consumption of foods high in sugar, carbohydrates, and fat.

- Choose lean proteins (e.g., fish, meat, eggs, milk, beans after fully cooked).

- Eat green, leafy vegetables.

- Eat fruits in two or three servings.

- Avoid alcohol and smoking.

\section{Exercise $^{6}$}

Regular physical activity is of great benefit to the general population and even more for people living with chronic conditions like diabetes and hypertension. Physical activity should be seen as a hobby and a valuable tool to overcome the monotony of the difficult confinement that many people around the world are currently experiencing. Public exercise facilities such as gyms, sports centers, and swimming pools have been closed too.

Below are a series of daily exercises that can be performed at home, provided by ANIAD, the Italian National Association of Athletes with Diabetes. The exercise intensity of each activity is comparable to 1 hour of brisk walking, resulting in an energy expenditure of 150 to $200 \mathrm{Kcal}$.

- Treadmill: 1-hour brisk walking (no need to run), which can also be split into three 20 -minute sessions. If possible, the slope should be adapted to individual fitness levels, to simulate an uphill walk.

- Stationary bicycle (either reclined or classic): two 15-minute sessions at variable intensity (if the equipment allows it). The sessions can be longer on a reclined bicycle since the effort is reduced by the backrest.

- Bodyweight exercises such as push-ups, squats, deep stationary lunges, sit-ups or crunches (to strengthen the abdomen), and forward flexes (to strengthen the lower-back muscles). These helps maintain muscle tone and, when performed correctly, can have excellent results.

- Joint mobility and stretching exercises that can be sourced from common workout, yoga, and pilates' routines.

Other ways to train at home:

- Walk up and down eight sets of stairs, for at least six floors. This is not recommended for people with T2DM who do not exercise regularly.

- Jump rope.

- Use small weights and home fitness accessories such as rubber bands, kettlebells, wrist weights, ankle weights, and pockets filled with heavy objects. Makeshift objects can also be used, such as buckets, cases, bottles filled with water or even small backpacks filled with objects of different weight.

These suggestions can be used to develop short, fragmented, or continuous training sessions. Here is an example of a series of "total body" exercises involving all main muscle groups, which anyone can do at home:

- Two series of 20 jumping jacks (onsite jumps with synchronized leg and arm spreading and closing).

- Two series of 15 crunches (abdomen strengthening). 
- Two series of 15 forward flexes (lower back muscle strengthening).

- Two series of 10 rowing exercises using dumbbells and slight forward flexion (back muscle strengthening).

- Two series of 8 push-ups (pectoral muscle strengtheningknees to floor for beginners).

- Two series of 8 sitting/standing hand-weight lifts (shoulder muscle strengthening).

- Five minutes of treadmill training or stationery/reclined bicycle.

- Three series $\times 15$ squats (lower limb strengthening).

- 20 minutes treadmill training.

- Final stretching and relaxation.

Make sure to avoid overload and adapt exercise intensity to individual ability and fitness level. It is also important to monitor your health before, during, and after exercising.

\section{Medications $^{5}$}

Strict compliance to medications is the utmost necessity to maintain target glycemic control. Inability to attend routine checkup, restricted availability of medications and checking blood glucose level from nearby laboratories are few of the common difficulties, being faced by most of our diabetic patients. Few general principles should be followed by every patient:

- Patients can be requested to check sugar at home as fasting and postprandial 2 hours with glucometer frequently-it is especially applicable with above target $\mathrm{HbA} 1 \mathrm{c}$ measured in last 3 to 4 months.

- Frequency of monitoring will be more in T1DM patients and T2DM patients on multiple subcutaneous insulin injections.

- Medications should not be stopped or changed to available one, without treating physician's advice.

- Sick day rule should be followed if any diabetic patient develops fever, running nose, cough, or shortness of breath apart from immediate visit to nearby hospital fever clinic.

- Time to time contact with treating physician to keep him/ her updated.

- Do not self-medicate with any medicines like hydroxychloroquine without prescription from a registered medical practitioner.

- Alert from hypoglycemia-do not fast for a long time; glucose tablets should be kept ready at home.

People living with diabetes should contact their healthcare team:

- If they vomit repeatedly (not able to hold down any food or drink for more than 6 hours), as they can quickly become very dehydrated.

- If their blood glucose stays high for more than 24 hours.

- If they develop symptoms that could be indicative of their developing diabetic ketoacidosis.

If a person with diabetes becomes ill, the following steps should be followed, even if the blood sugar levels are within the target range:
- Take diabetes medication as usual. Insulin treatment should never be stopped.

- Test blood glucose every four hours, and keep track of the results.

- Drink extra (calorie-free) fluid, ${ }^{*}$ and try to eat as normal.

- Weigh yourself every day. Losing weight while eating normally is a sign of high blood glucose.

- Check temperature every morning and evening. A fever may be a sign of infection.

- Ideal blood sugar levels should be between 6 and $10 \mathrm{mmol} / \mathrm{L}$ (110-180 mg/dL).

- For T1DM patients-blood glucose levels should be checked at least every 4 hours or frequently if necessary (every 2 hours if ketone present in urine)

- For T2DM patients on insulin-if the blood glucose levels stay above $10 \mathrm{mmol} / \mathrm{l}(180 \mathrm{mg} / \mathrm{dL})$, they should increase their insulin dose. Extra blood glucose testing will often be necessary. Testing should be done every 4 hours, especially if the blood glucose levels are high (over $15 \mathrm{mmol} / \mathrm{L}$ [270 mg/dL]).

*Drink plenty of fluids-120 to $180 \mathrm{~mL}$ every half an hour to prevent dehydration.

It might also be necessary to drink sugary beverages if it is not possible to take in $50 \mathrm{~g}$ of carbohydrates through food. The amount of sugary beverages should nevertheless be carefully controlled to prevent blood sugar levels from rising too much.

- Sick day rule in detail is available online at IDF site for people with diabetes. ${ }^{7}$

\section{Conclusion}

COVID-19 has forever changed the way we live. We have come to understand and implement personal and social hygiene in a better way. Social distancing is important not only to save the vulnerable people like people living with diabetes from COVID-19 infection but also to prevent the diabetes outpatient services from becoming hotspots of disease spread. Telemedicine is an important technologic help of bringing together the patient and the doctor ensuring an optimal level of diabetes care in a large majority of patients living with diabetes. For patients needing visit to clinic/hospital, it is important to make the clinic/hospital COVID-19 safe to prevent the spread of COVID-19 among health-care staff and patients.

\section{Funding}

None.

\section{Conflict of Interest}

None declared.

\section{References}

1 Wu Z, McGoogan JM. Characteristics of and Important Lessons From the Coronavirus Disease 2019 (COVID-19) Outbreak in China: Summary of a Report of 72314 Cases From the Chinese Center for Disease Control and Prevention. JAMA 2020;323(13):1239-1242 
2 Yang $\mathrm{X}, \mathrm{Yu} \mathrm{Y}, \mathrm{Xu}$ J, et al. Clinical course and outcomes of critically ill patients with SARS-CoV-2 pneumonia in Wuhan, China: a single-centered, retrospective, observational study. Lancet Respir Med 2020;8(5):475-481

3 Guan WJ, Ni ZY, Hu Y, et al; China Medical Treatment Expert Group for Covid-19. Clinical characteristics of coronavirus disease 2019 in China. N Engl J Med 2020;382(18):1708-1720

4 Telemedicine Practice Guidelines. https://www.mohfw.gov.in/ pdf/Telemedicine.pdf. Accessed July 31, 2020
5 COVID-19 and diabetes. https://www.idf.org/aboutdiabetes/ what-is-diabetes/covid-19-and-diabetes.html . Accessed July 31,2020

6 Healthy nutrition and home-based exercise. https://www.idf. org/aboutdiabetes/what-is-diabetes/covid-19-and-diabetes/ home-based-exercise.html. Accessed July 31, 2020

7 How to manage diabetes during an illness? https://www. idf.org/component/attachments/?task=download\&id=2155: IDFE-Sick-day-management. Accessed July 31, 2020 